\title{
RESIDUAL STRESS CONTROL FOR SUPERALLOYS DISK COOLING TREATMENTS
}

\author{
Zhong-nan BI, Chao TANG, Jing-long QU, Jin-hui DU, Ji ZHANG \\ Central Iron and Steel Research Institute, Beijing, China; 100083
}

Keywords: Residual stress, Disk, Cooling treatment

\begin{abstract}
The cooling method is a major factor in controlling residual stress, but also has significant influence on $\gamma^{\prime}$ precipitation behavior and thereby mechanical performance. These effects of cooling rate make residual stress control a more complicated issue for superalloys. Residual stress evolution in a $\varphi 150 \mathrm{~mm} 720 \mathrm{Li}$ disk during different cooling treatments has been studied by FEM simulation. XRD measurements and contour method were also carried out on the heat treated disk to verify the simulation predictions. Considering the influence of cooling type on residual stress and $\gamma^{\prime}$ precipitation behavior, a special solid quenching treatment using induction heated dies as heat-transfer medium was introduced to relieve the residual stress and ensure the strength simultaneously.
\end{abstract}

\section{Introduction}

Residual stress is that which remains in a body that is stationary and at equilibrium with its surroundings [1]. Due to the uneven plastic deformation, residual stress is usually inevitable to be introduced during processing for forgings. The precise residual stress distribution is of importance to engine manufacturers for two distinct reasons [2, 3]. Firstly, machining processes remove constraining material, which causes the component to distort as the residual stresses re-distribute to reach a new equilibrium state. If unaccounted for, such distortions can lead to significant re-working of the component, or additional machining operations. Secondly, residual stresses have a direct impact on the performance of a component as they add to the applied stresses during operation. In the case of tensile residual stresses, they can deteriorate fatigue life. Furthermore, release of residual stress by creep will affect the structural stability of the components during operation. These affects will ultimately pass on to component life predictions for service. For these reasons, it has become increasingly important to predict and control the residual stresses. 
Generally, residual stresses in superalloys disk components originate from heat treatments and are produced when the material yields locally during post solution fast cooling. The quenched-in residual stresses are then relaxed during aging, but not eliminated [4-7]. Therefore, it is meaningful to relieve the residual stress formed during quenching. Cracking may also occur when the stress is too high or non-uniform. It is well known that the cooling process after solution also has significant influence on $\gamma^{\prime}$ precipitation behavior and mechanical performances. Therefore, the residual stress control is more complicated for superalloys, especially for the ones with high alloying level.

In the present paper, the evolution behavior of residual stress for $720 \mathrm{Li}$ disk during different cooling treatments was studied by FEM simulation. XRD measurements and contour method were also carried out to verify the simulation predictions. Through consideration of the influence of cooling method on residual stress and tensile stress, a technique was selected to relieve the residual stress and ensure the mechanical properties simultaneously.

\section{Experimental Procedure}

As a typical disk used superalloy, 720Li alloy was selected as the study material in this study [8]. The nominal chemical composition of $720 \mathrm{Li}$ alloy is given in Table 1.

Table 1 Chemical composition of the investigated 720Li ingot (wt. \%)

\begin{tabular}{cccccccccccc}
$\mathrm{C}$ & $\mathrm{S}$ & $\mathrm{P}$ & $\mathrm{Cr}$ & $\mathrm{W}$ & $\mathrm{Mo}$ & $\mathrm{Al}$ & $\mathrm{Ti}$ & $\mathrm{Co}$ & $\mathrm{B}$ & $\mathrm{Zr}$ & $\mathrm{Ni}$ \\
\hline & & & & & & & & & & & \\
0.017 & 0.001 & 0.005 & 15.98 & 1.19 & 2.98 & 2.75 & 4.91 & 14.86 & 0.017 & 0.025 & $\mathrm{Bal}$ \\
\hline
\end{tabular}

The evolution behavior of residual stress for $\$ 150 \mathrm{~mm} \times 60 \mathrm{~mm} 720 \mathrm{Li}$ disk (pancake) during different cooling treatments was studied by FEM simulation using deform 2D V9.0 software. Such modeling processes are becoming mature enough to predict the residual stress of the component [9] with improved accuracy.

Oil quenching $(O Q)$ and a special solid quenching of the disk were practiced respectively. Residual stress measurements using XRD and contour method were used to verify the simulation predictions. Yield strength of the disk at different locations was also predicted and tested respectively for optimized cooling treatment. All the heat treatments are based on the accepted method for this alloy. The solution treatment is $1100^{\circ} \mathrm{C} / 4 \mathrm{~h}$ (with different cooling method); ageing 


\section{Results and Discussion}

\section{Effect of cooling rate on Residual stress}

The evolution of temperature distribution for the investigated 720Li disk is shown in Figure 1 . Because of the volume difference due to different local temperatures, thermal stress are generated. As shown in Fig.1, the hoop and radial thermal stress transformed from inside-tensile/ outside-compressive to inside-compressive/ outside-tensile. The magnitude of axial stress is not as high as the hoop and radial stresses.
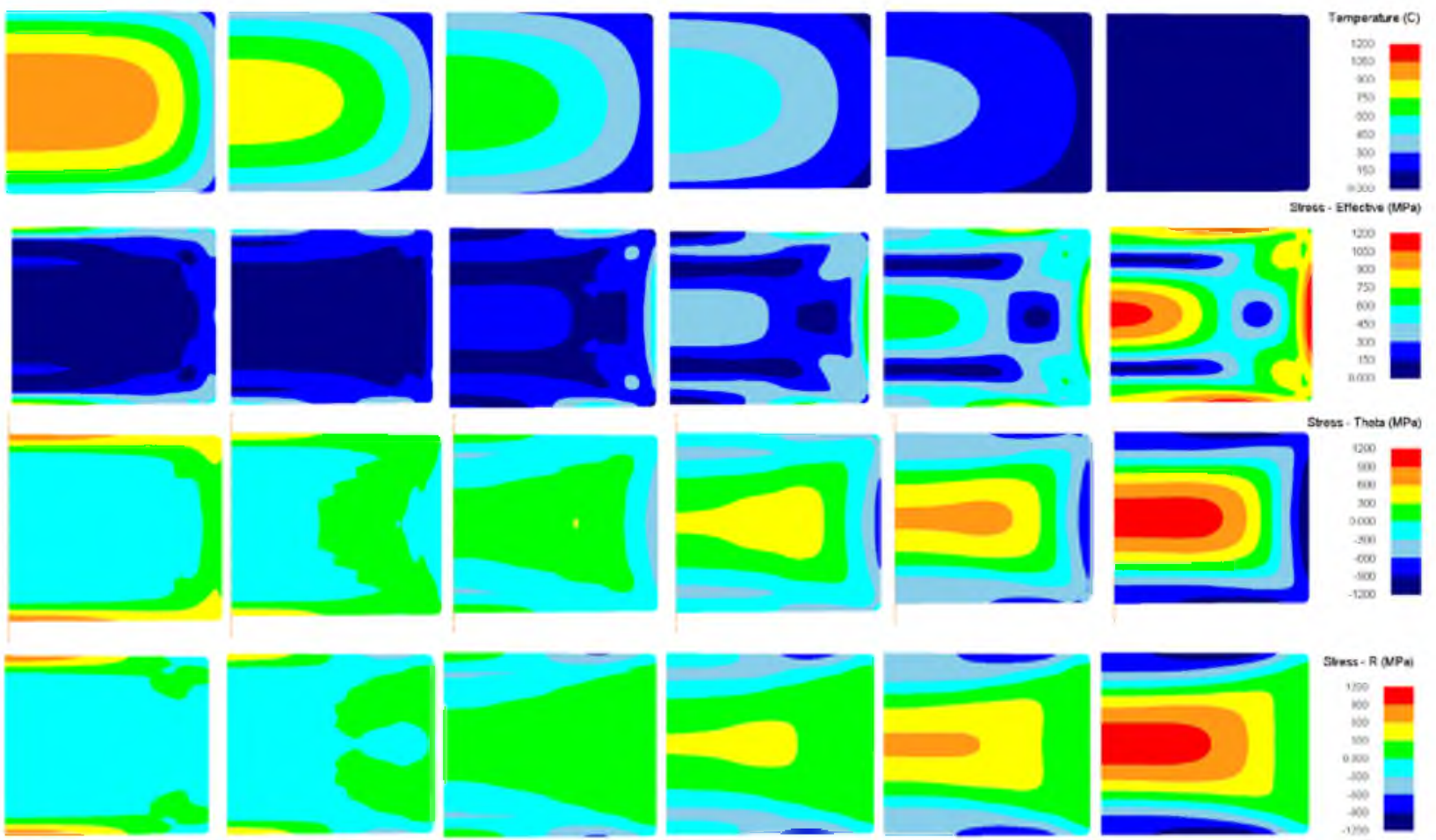

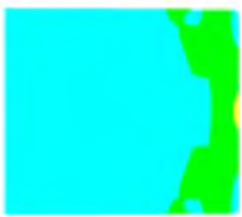

$50 \mathrm{~s}$

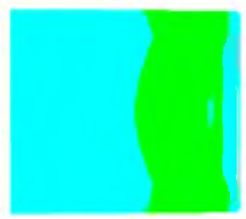

$100 \mathrm{~s}$

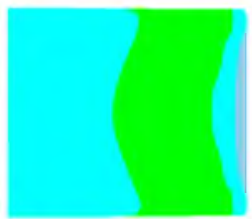

$150 \mathrm{~s}$

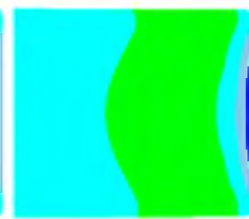

$200 \mathrm{~s}$

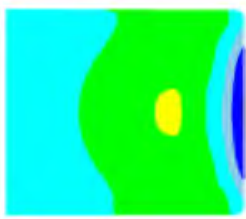

$300 \mathrm{~s}$

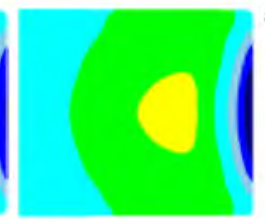

$600 \mathrm{~s}$

Figure 1 Calculated temperature and thermal stress transformation during OQ of the $720 \mathrm{Li}$ disk

In order to describe the stress level with a single value, DEFORM uses the Von Mises stress to define the characteristic "effective stress". The effective stress is defined as $\bar{\sigma}=\sqrt{\left(\sigma_{1}-\sigma_{2}\right)^{2}+\left(\sigma_{2}-\sigma_{3}\right)^{2}+\left(\sigma_{3}-\sigma_{1}\right)^{2}} / \sqrt{2}$ 
Where $\sigma_{1}, \sigma_{2}$ and $\sigma_{3}$ are the principal stresses. For most metals, the peak effective stress is indicative of the onset of plastic flow [9]. Figure 1 shows the rise in stress with temperature drop.

The maximum value of effective residual stress at RT is nearly $1050 \mathrm{MPa}$ for the investigated 720Li disk after OQ. As shown in the Figure 2, decreasing of cooling rate can lower the residual stress magnitude After air cooling (AC), the maximum value of effective residual stress is as lower as only $150 \mathrm{MPa}$.

(a)

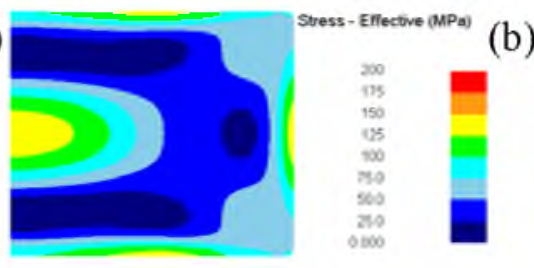

(b)

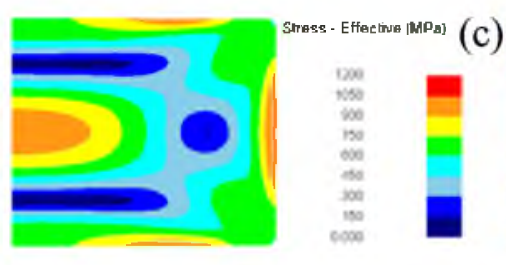

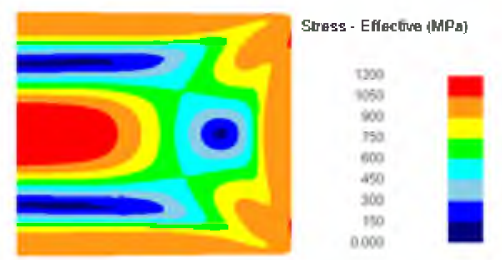

Figure 2 Calculated effective residual stress after quenching from $1100^{\circ} \mathrm{C}$ to room temperature with different cooling mediums (a) AC; (b) OQ; (c) WQ (water quenching)

\section{Effect of cooling rate on $\gamma^{\prime}$ and strength}

It is widely known that the cooling process after solution treatment also has significant influence on $\gamma^{\prime}$ precipitation behavior which will strongly affect the mechanical performances of $720 \mathrm{Li}$ alloy [10-12]. Microstructure observation and tensile test (after standard heat treatment) of 720Li alloy with different cooling rates was carried out. As shown in Figure 3, the volume and size of $\gamma^{\prime}$ varies with cooling rates; especially for the secondary $\gamma^{\prime}$. Consequently, the yield strength at room temperature (RT) falls with lower cooling rate after solution. Further more, it is reported that the affecting temperature region for secondary $\gamma^{\prime}$ is approximately $800 \sim 1000^{\circ} \mathrm{C}[11,12]$.

(a)

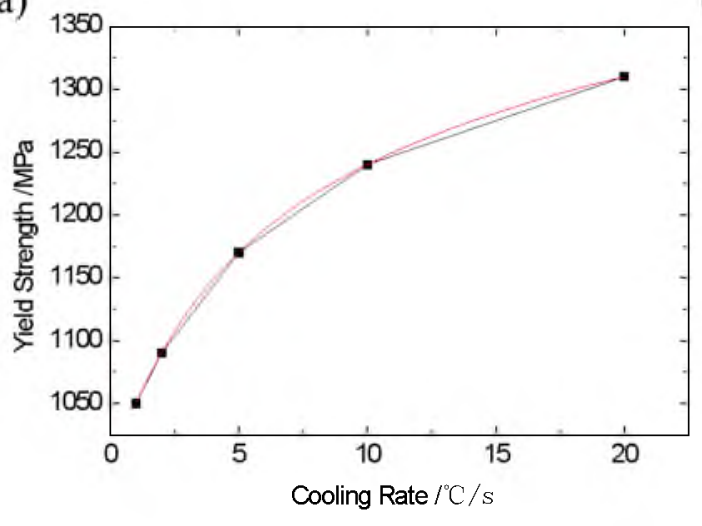

(b)

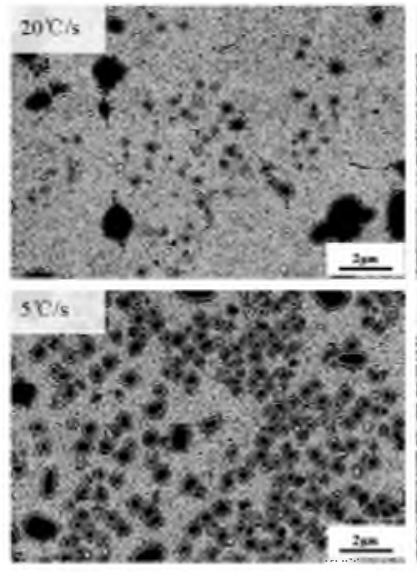

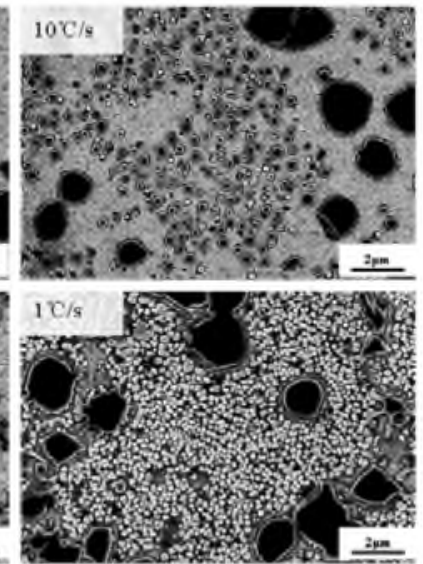

Figure 3 Effect of cooling rate on (a) RT tensile strength and (b) microstructure for 720Li alloy 
By nonlinear fitting using Origin 8.0, a mathematical relation between cooling rate and yield stress has been found. Applying the relationship through Deform 2D an approximation of the yield strength at different location can be predicted. The simulation results shown in Figure 4 demonstrate a decrease in cooling rate reduces the yield strength of $720 \mathrm{Li}$. Simultaneously the disk residual stress magnitude also decreases.

In addition, for an OQ or WQ disk, the predicted strength at the central position is lower than the ones at outer position as a result of the lower local cooling rate.

(a)

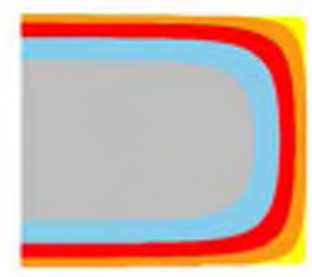

(b)

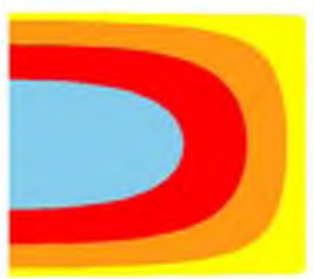

(c)

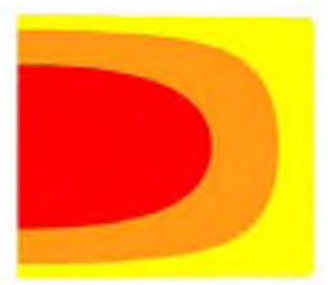

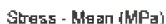

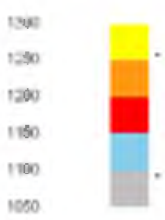

Figure 4 Predicted RT tensile strength of the investigated 720Li disk with different cooling ways (a) AC; (b) OQ; (c) WQ

Finding the wav for residual stress relieving

As indicated decreasing the cooling rate of the $720 \mathrm{Li}$ disk will reduce the residual stress and tensile strength simultaneously. It is necessary to find an alternative method to reduce residual stress.

(a)

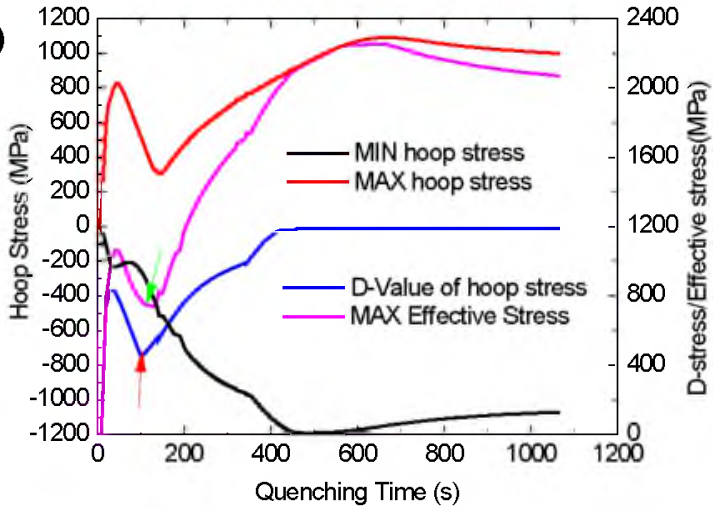

(b)

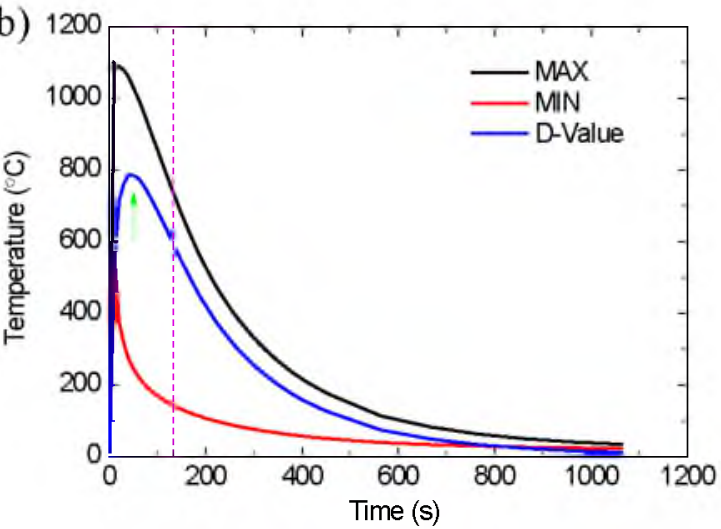

Figure 5 Calculated (a) stress and (b) temperature transformation during $O Q$ for the investigated $720 \mathrm{Li}$ disk

As shown in Figure 1, the thermal stress changes direction during quenching. Therefore a valley value when the direction change exists. For hoop stress, after quenching for about 130s, the $\mathrm{D}$-value of maximum and minimum hoop stress reached an inflexion point, shown in Figure 5a 
(marked by green arrow). At approximately the same time, the effective stress also reaches a low inflection point (marked by red arrow).

Therefore consideration was given to lowering the cooling rate after "the low stress point" to decrease the residual stress for the disk. As the maximum temperature of the disk has dropped under $800^{\circ} \mathrm{C}$, after 130 s for OQ (Figure $5 \mathrm{~b}$ ), the secondary $\gamma^{\prime}$ and strength are largely unaffected. However, it was found that the effect of these cooling-adjusting for decreasing residual stress are limited as illustrated in Figure 6(b, c, d).

It was found that "the largest temperature difference" (as marked by green arrow in Figure 5b) rises to the peak point after quenching for $45 \mathrm{~s}$, which is achieved much earlier than "the low stress point". As shown in Figure 6, the simulated results of more cooling conditions, it is believed that "the largest temperature difference" is the main affecting factor for residual stress. Therefore once the largest temperature difference is formed, there is no effective way to change the final residual stress. For this reason the plans around the "low stress point" is ineffective.

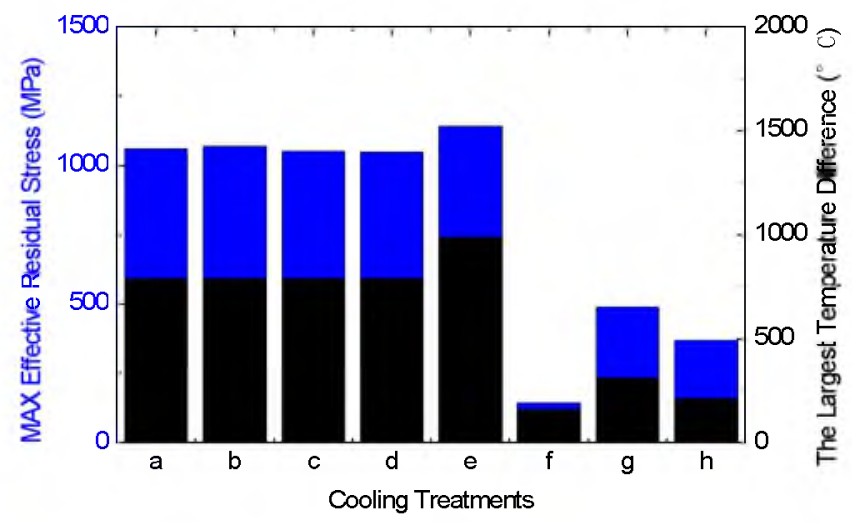

From: $1100^{\circ} \mathrm{C}$

a: $\mathrm{OQ}$ to $20^{\circ} \mathrm{C}$;

b: $\mathrm{OQ} / 130 \mathrm{~s} \rightarrow \mathrm{WQ}$ to $20^{\circ} \mathrm{C}$;

c: $\mathrm{OQ} / 130 \mathrm{~s} \rightarrow \mathrm{AC}$ to $20^{\circ} \mathrm{C}$;

d: $\mathrm{OQ} / 130 \mathrm{~s} \rightarrow$ heat to $600^{\circ} \mathrm{C}$ and hold for $600 \mathrm{~s} \rightarrow \mathrm{AC}$ to $20^{\circ} \mathrm{C}$;

e: WQ to $20^{\circ} \mathrm{C}$;

f: $\mathrm{AC}$ to $20^{\circ} \mathrm{C}$ :

g: $\mathrm{CC}$ to $600^{\circ} \mathrm{C} \rightarrow \mathrm{AC}$ to $20^{\circ} \mathrm{C}$;

h: $\mathrm{CC}$ to $800^{\circ} \mathrm{C} \rightarrow \mathrm{AC}$ to $20^{\circ} \mathrm{C}$.

$* \mathrm{CC}=$ Control Cooling,

Convection coefficient $=1 \mathrm{~N} / \mathrm{s} / \mathrm{C} / \mathrm{mm}$

Figure 6 Maximum effective stress (blue blocks) and "the largest temperature difference" (black blocks) for the investigated 720Li disk with various cooling treatments.

Technically, decreasing heat transfer between the work piece and environment or increasing the temperature of the cooling medium can both lower "the largest temperature difference" and decrease the residual stress. But lower heat transfer in the high temperature range $\left(800 \sim 1000^{\circ} \mathrm{C}\right)$ will affect the fraction of secondary $\gamma^{\prime}$ and lower the tensile strength (as shown in Figure 4). As shown in Figure $6 \mathrm{~g}$, $\mathrm{h}$, after control cooling to higher temperature such as $600^{\circ} \mathrm{C} \mathrm{\&} 800^{\circ} \mathrm{C}$ with a reasonable high cooling rate, and then $\mathrm{AC}$ to room temperature, much lower residual stress is achieved. Further more, the residual stress is lower with higher medium temperature.

\section{Special solid quenching treatment}

Based on the simulation results above, a special solid quenching treatment was developed for the 
720Li disk. The solid quench is achieved by using the dies in an isothermal forging press as shown in figure 7. Die temperature was controlled by induction heating. It is well known that the heat transfer coefficient between metals in good contact is as large as $10^{3} \sim 2 \times 10^{4} \mathrm{~W} / \mathrm{K} / \mathrm{m}^{2}$, that may be larger than salt-melted quenching, gas cooling and even water or oil quenching. In order to increase the heat transfer coefficient, proper load is promoted by the press. As tested by using a work piece instrumented with thermocouples, the heat transfer coefficient can reach about $10^{4}$ $\mathrm{W} / \mathrm{K} / \mathrm{m}^{2}$ with applied pressure due to the larger micro- contact surface.

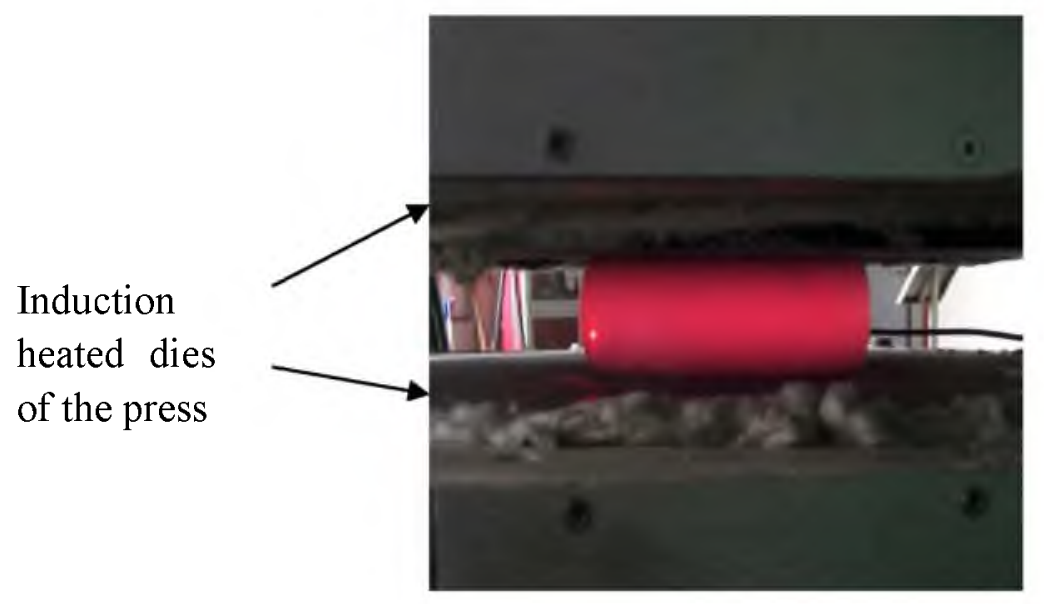

Figure 7 Illustration of "Special solid quenching treatment"

(a)

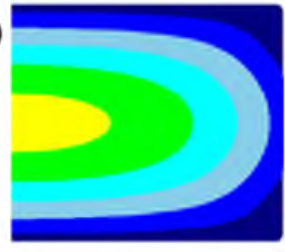

(b)

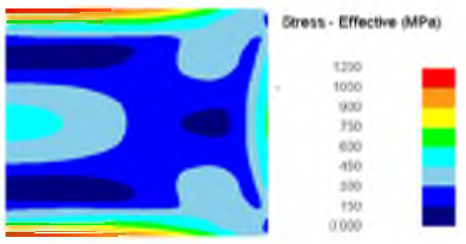

(c)

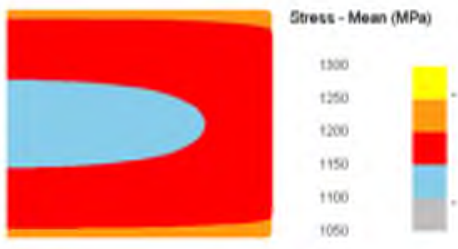

Figure 8 Calculated (a) temperature distribution, (b) effective residual stress and (c) the yield strength of the investigated $720 \mathrm{Li}$ disk after "special solid quenching" then AC to RT

As shown in Figure 8a, after the "Special solid quenching" for over $300 \mathrm{~s}$ with $600^{\circ} \mathrm{C}$ die temperature, the maximum temperature of the disk is lower than $700^{\circ} \mathrm{C}$, at which the $\gamma^{\prime}$ is less affected; and then AC to room temperature, lower residual stress was achieved without obviously losing strength properties as shown in Figure 8b, c. Comparing to the results with OQ as shown in Figure $2 \mathrm{~b}$ and Figure $4 \mathrm{~b}$, the effective residual stress at central position is reduced from $1050 \mathrm{MPa}$ to about $500 \mathrm{MPa}$, though the effective residual stress at the contact faces is still high (the component is unaffected); In addition, the predicted yield strength is not lowered significantly, but more is uniform. 


\section{Verification}

In order to verify the simulated results above, residual stress measurements using XRD and contour method were carried out respectively. Yield strength of the disk at different locations was also tested. The sketch map for the positions of residual stress measurement and tensile test sampling is shown in Figure 9. P1, P2, P3 are surface residual stress measurement position using XRD (Hoop stress and Radial stress, mid-value and errors of 6 measured values); P4, P5, P6 are inside residual stress measurement position using contour method (details on the contour method are given in [3]); S1, S2, S3, S4, S5, S6 are sampling position for tensile tests (in the tangential direction).

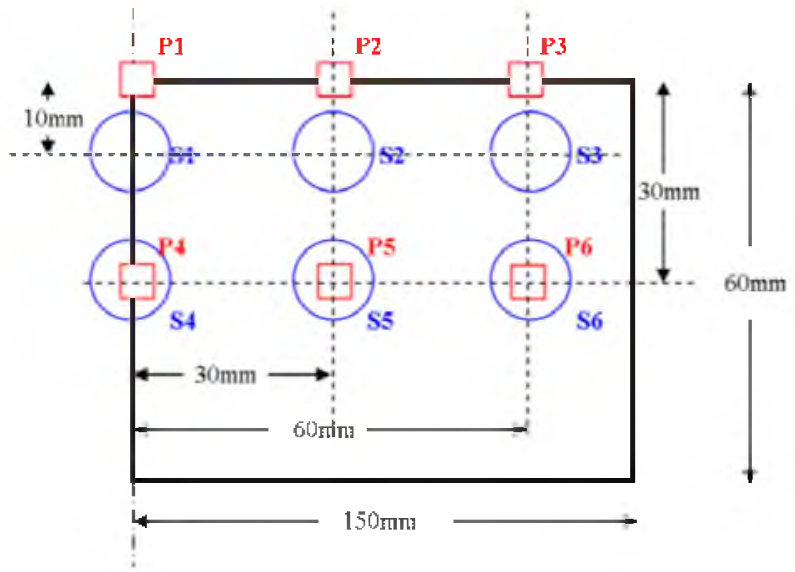

Figure 9 Sketch map for the positions of residual stress measurement (P1 P6) and tensile test sampling (S1 S6)

OQ and a "special solid quenching" of the disk were practiced respectively. Figure 10 presents a comparison of the predicted and measured values for the residual stress and yield strength. The predicted values are in good agreements with the measured results.

(a)

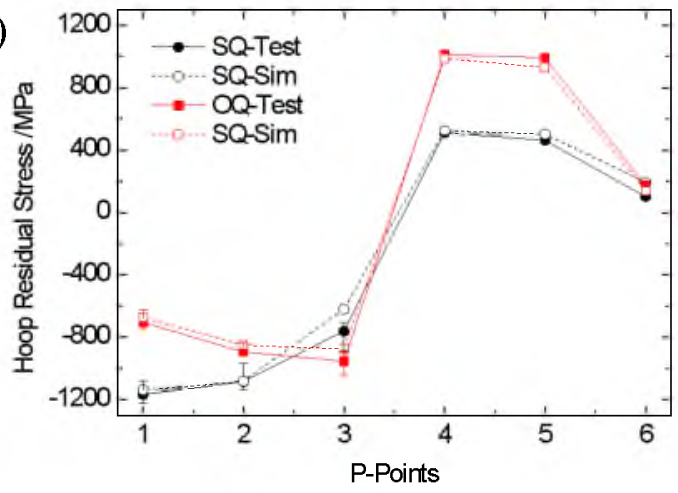

(b)

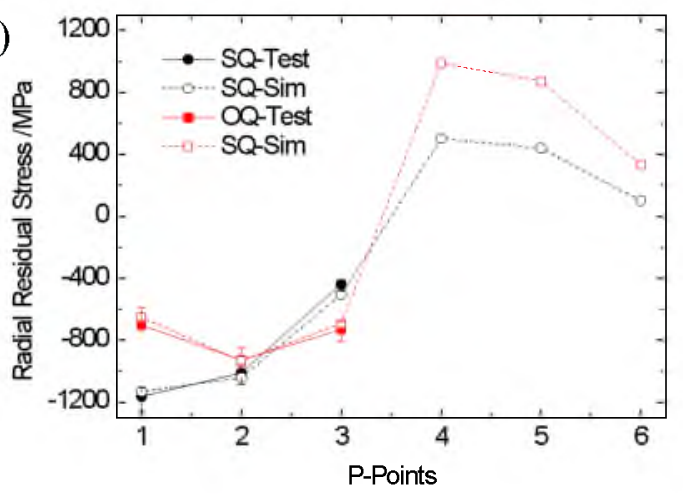




\section{(c)}

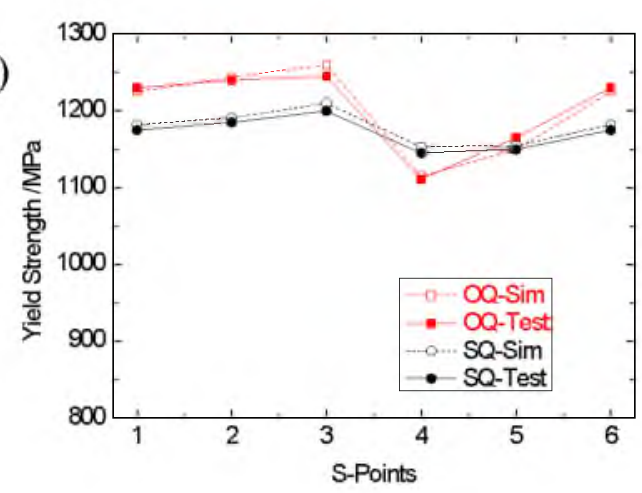

Figure10 (a) Measured and simulated (b) residual stress and (c) yield strength at different locations of the investigated 720Li disk with OQ and "Special solid quenching" respectively

\section{Discussion}

The method introduced in the present paper is a method to reduce residual stress of the superalloy forgings. For ordinary disk forgings, reducing the thickness as much as possible can also relieve residual stress due to the decreased temperature difference of the disk during cooling. Meanwhile, the tensile strength is ensured because of the cooling rate.

It should be noted that the distribution characteristic of the residual stress was also changed due to the different temperature field developed by changing the cooling media changed from liquid immersion to contacted solid end faces. Further more, precise designed residual stress can be introduced by controlled cooling treatment. Optimized residual stress could maximize the disk performance during operation.

\section{Conclusion}

Cooling process is very important for residual stress controlling. Decreasing the cooling rate after solution can lower the residual stress magnitude of the investigated 720Li disk. However, the amount and size of $\gamma^{\prime}$ varies with cooling rate and the yield strength decreases with lower cooling rate. It is found that "the largest temperature difference" is the main affecting factor for residual stress. So the residual stress can also been lowered with elevated medium temperature. A special solid quenching treatment using induction heated dies as heat-transfer medium was applied to reduce the residual stress and simultaneously ensure the mechanical properties. In order to increase the cooling rate during the high temperature region and promote tensile strength, proper 
pressure was applied to increase the heat transfer coefficient between disk and dies.

\section{References}

1. P. J. Withers and H. K. D. H. Bhadeshia, "Residual stress Part 1 - Measurement techniques," Materials Science and Technology, 17(2001), 355-365.

2. D. L Ball, B. K Tom, R. J. Bucci, and M. A James, "Toward Understanding the Impact of Bulk Residual Stress on the Life, Weight and Cost of Primary Aircraft Structure". (Paper presented at the Residual Stress Summit, Tahoe City, CA, 28 September 2010).

3. J. Rolph, M. Preuss, N. Iqbal, M. Hofmannm, S. Nikov, M. C. Hardy, M. G. Glavicic, R. Ramanathan, and A. Evans, "Residual stress evolution during the manufacture of aerospace forgings," Superalloys 2012, ed. E.S. Huron, et al (Warrendale, PA: TMS, 2012), 881-891.

4. R. A. Wallis, "Modeling of Quenching, Residual Stress Formation, and Quench Crack," Metal Process Simulation, ed. D. U. Furrer, and L. S. Semiatin (ASM International: TMS, 2010), 547-585.

5. J. M. Franchet, F. Devy, P. E. Mosser, Y. Honnorat, and A. Benallal, "Modeling during the Oil Quenching of an Astroloy Turbine Disk," Superalloys 1992, ed. D. Kissinger, and D.L. Klarstrom (Warrendale, PA: TMS, 1992), 73-82.

6. G. S. Shen, N. Cooper, N. Ottow, R. Goets, and J. Matlik, "Integration and Automation of Residual Stress and Service Stress Modeling for Superalloy Component Design," Superalloys 2012, ed. E.S. Huron, et al (Warrendale, PA: TMS, 2012), 129-134.

7. D. U. Furrer, R. Shankar, and C. White, "Optimizing the Heat Treatment of Ni-Based Superalloy Turbine Discs," JOM, 2003, 32-34.

8. D. U. Furrer, and H. Fecht, "Ni-based Superalloys for Turbine Discs," JOM, 1999, 14-17.

9. DEFORM, trademark of Scientific Forming Technologies Corporation, Columbus, $\mathrm{OH}$.

10. M. P. Jackson, R. C. Reed, "Heat Treatment of UDIMET 720Li: the Effect of Microstructure on Properties," Materials Science and Engineering, A259 (1999), 85-97.

11. J. Mao, K. M. Chang, W. H. Yang, K. Ray, S. P. Vaze and D. U. Furrer, "Cooling Precipitation and Strengthening Study in Powder Metallurgy Superalloy U720Li," Metallurgical and Materials 
Transaction, A32(2001), 2441-2452.

12. C. Tang, "Study on The Relationships among Solution Heat Treatment, Microstructure and Mechanical Properties in GH720Li Alloy," (Master. thesis, Central Iron and Steel Institute, 2013), $56-60$. 